\title{
An alternative to "brutacaine": a comparison of low dose intramuscular ketamine with intranasal midazolam in children before suturing
}

\author{
R G McGlone, S Ranasinghe, S Durham
}

\begin{abstract}
Objective-To compare the use of low dose intramuscular ketamine with high dose intranasal midazolam in children before suturing.

Methods-Altogether 102 children with simple wounds between 1 and 7 years old were allocated to the two study groups.

Results-Two children were excluded from the study because of deviation from the agreed protocol. The $\mathbf{5 0}$ children in the ketamine group were less likely to cry or need to be restrained during the procedure than those in the midazolam group $(\mathbf{p}<0.01)$.The median oxygen saturation was $97 \%$ in both groups. There was no difference in the recovery behaviour and the range of time at which children were ready for discharge, although the median time for the latter was shorter in the midazolam group (75 $v 82$ minutes). Vomiting occurred in nine of the ketamine and four of the midazolam group. After discharge both groups had an unsteady gait $(73 \% v$ $71 \%$ ) which usually resolved within two hours.

Conclusion-Intranasal midazolam (0.5 $\mathrm{mg} / \mathrm{kg}$ ) effectively sedated the children in that none could remember the suturing. However a significant number still had to be restrained $(86 \% v 14 \%)$. Intramuscular ketamine $(2.5 \mathrm{mg} / \mathrm{kg})$ produced dissociative anaesthesia in the majority of cases and was the preferred drug of nurse, doctor, and parent.

(F Accid Emerg Med 1998;15:231-236)
\end{abstract}

Keywords: suturing; children; ketamine; midazolam

Accident and

Emergency

Department, Royal

Lancaster Infirmary,

Ashton Road,

Lancaster LA1 3ES

R G McGlone

$S$ Ranasinghe

S Durham

Correspondence to:

Dr McGlone.

Accepted for publication 4 March 1998 been used whereby children have "been wrapped up in a blanket with or without a premedication. The suturing is done with the aid of local anaesthetic, but the whole process can be terrifying for the child and suturing a moving target inevitably gives a poor cosmetic effect. Some children will cooperate with suturing, if time is spent explaining the procedure and distraction techniques are used. However many remain terrified and for these children future visits to the $\mathrm{A} \& \mathrm{E}$ department or general practitioner's surgery often precipitate screams of protestation. Surely there must be a better way?

Two techniques for sedation using intranasal midazolam and intramuscular ketamine are already used in this department by senior doctors. They have an established role for this particular clinical problem and have been extensively used in the United States. ${ }^{1-8}$ We proposed to compare the two techniques.

Ketamine was introduced into general clinical practice in 1970. It generates a functional and electrophysiological dissociation between the cortical and limbic systems providing a "dissociative anaesthesia". Ketamine preserves laryngeal reflexes and muscle tone is not inhibited. The dissociated state induced by ketamine can't be equated with the typical general anaesthetic because protective airway reflexes are maintained without clinical respiratory depression.

Although the intranasal route has been used, ${ }^{10}$ we choose to use the intramuscular route to ensure that the exact dose was given as inevitably some of the intranasal dose would be swallowed. We proposed to use ketamine 2.5 $\mathrm{mg} / \mathrm{kg}$ with atropine. The latter was used to reduce the excessive salivation and lacrimation which can be a feature of ketamine. By using this lower dose we hoped to reduce the incidence of vomiting (10\%) and shorten the recovery time (80 minutes). ${ }^{1}$ A previous study showed that lower doses were associated with fewer side effects. ${ }^{11}$

Intranasal midazolam (intravenous preparation) has a short onset of action of five to 10 minutes. ${ }^{4}$ We proposed to use a dose of 0.5 $\mathrm{mg} / \mathrm{kg}$ because a previous study showed that such a dose was more likely to provide adequate sedation yet with no significant oxygen desaturation. ${ }^{6}$ 
Table 1 Characteristics of patients and their wounds; values are mean (range) unless otherwise stated

\begin{tabular}{lcc}
\hline & Ketamine & Midazolam \\
\hline Age (years) & $36(1-7)$ & $3.6(1-7)$ \\
Weight (kg) & $17.3(9-29)$ & $17.4(10-25)$ \\
No of facial wounds & 35 & 33 \\
Size of wound (cm) & $2.3(1.3-6)$ & $2.1(1.2-7)$ \\
No closed in one layer & 31 & 32 \\
No of sutures & $5.5(2-16)$ & $5.1(2-10)$ \\
\hline
\end{tabular}

\section{Patients and methods}

PATIENTS

Children between 12 months and 7 years who presented with lacerations were studied. The exclusion criteria were: (1) The laceration was complicated by more serious injury such as bone fracture or a closed head injury associated with loss of consciousness. (2) There was a current upper respiratory tract infection or active asthma. (3) A full meal had been eaten within three hours. (4) The child had severe cognitive and/or motor delay. (5) Hypertension, glaucoma, severe behavioural problems, or porphyria were present. (6) There was a prior adverse reaction to ketamine.

PROCEDURE

Excluded patients were sutured under local anaesthetic, except for those having recently eaten who were asked to either wait or attend the department later. After informed consent from the parent, the child was allocated for treatment alternating between the midazolam group or the ketamine group. All children had the procedure done by a senior $A \& E$ doctor. Parents were given a fact sheet on the drug given, which included guidance for when the child was discharged.

The midazolam group had $0.5 \mathrm{mg} / \mathrm{kg}$ ( 5 $\mathrm{mg} / \mathrm{ml}$ ) intranasally (dose split between either nostril) with head of trolley tilted down or sat back on parent's lap with head down. The dose was drawn up in a $2 \mathrm{ml}$ syringe and administered slowly by the doctor. Infiltration of local anaesthetic was done after at least 10 minutes had elapsed.

The ketamine group had $2.5 \mathrm{mg} / \mathrm{kg}$ (100 $\mathrm{mg} / \mathrm{ml}$ ) drawn up in a $1 \mathrm{ml}$ syringe, then the appropriate dose of atropine $(0.01 \mathrm{mg} / \mathrm{kg}$ maximum $0.3 \mathrm{mg}$ ) was drawn up into the same syringe from an ampoule of atropine $(0.5 \mathrm{mg}$ in $1 \mathrm{ml}$ ). The intramuscular injection was into the vastus lateralis (lateral thigh). The child could be held in the arms of the parent if appropriate. Infiltration of local anaesthetic occurred after at least five minutes had passed. If sedation was not achieved by 10 minutes then a further dose of ketamine $1 \mathrm{mg} / \mathrm{kg}$ excluding atropine could be given.

The procedure took place in the $A \& E$ theatre with resuscitation equipment available.
After the child's drug had been administered he or she was transferred to a trolley and a pulse oximetry probe was attached to a digit. Parents were encouraged to stay with their child during the procedure.

During recovery the child was placed on his or her side. In both groups the lighting was reduced to one main light and the area was kept as quiet as possible. The latter precautions were to help reduce the incidence of dysphoric reactions. The child was observed until they returned to their baseline level of awareness and verbalisation.

\section{OBSERVATIONS}

During the procedure oxygen saturation was monitored continuously, the lowest oxygen saturation on the trend graph (on the Propaq display) was documented.

Behaviour before the procedure, during the infiltration of local anaesthetic, and during suturing was rated on a four point scale by the nurse as either: (0) cooperative or sleeping; (1) intermittent crying or fighting; (2) continuous crying or fighting; or (3) uncontrolled crying or fighting.

If any restraint was needed the nurse would document whether head, arms, and/or legs were restrained and coded 0 to 3 depending on how many were restrained. Reaction of parents during procedure was documented as either showing no response or feeling faint.

Other observations included occurrence and frequency of vomiting, lacrimation, salivation, and rashes. If any respiratory difficulty occurred then this was documented along with any intervention.

Difficulty in administration of the intranasal midazolam was recorded.

The doctor recorded the site of the laceration, the length, the number of sutures, and whether it was closed in one layer.

During recovery the behaviour of the child was documented by the nurse on a four point scale: (0) quiet and uneventful; (1) mild agitation; (2) moderate agitation; or (3) pronounced agitation.

DISCHARGE CRITERIA

The child had to be able to recognise his or her parents, demonstrate purposeful motor activity, and be able to walk unaided. Parents were instructed that their child should not walk independently for the first two hours after discharge and only clear fluids should be allowed during this time.

\section{FOLLOW UP}

Parents were contacted by telephone by medical staff within 24 to 72 hours to complete a

Table 2 Behaviour of children expressed on four point scale; values are number (\%) of patients

\begin{tabular}{|c|c|c|c|c|c|c|}
\hline \multirow[b]{2}{*}{ Behaviour } & \multicolumn{2}{|c|}{ Before procedure } & \multicolumn{2}{|c|}{ During anaesthetic } & \multicolumn{2}{|c|}{ During suturing } \\
\hline & Ketamine & Midazolam & Ketamine & Midazolam & Ketamine & Midazolam \\
\hline Cooperative & $20(40)$ & $30(60)$ & $38(76)$ & $7(14)$ & $46(92)$ & $20(40)$ \\
\hline Intermittent crying & $23(46)$ & $14(28)$ & $10(20)$ & $12(24)$ & $4(8)$ & $15(30)$ \\
\hline Continuous crying & $4(8)$ & $4(8)$ & $2(4)$ & $24(48)$ & 0 & $7(14)$ \\
\hline Uncontrolled crying & $3(6)$ & $2(4)$ & 0 & 7 (14) & 0 & $8(16)$ \\
\hline
\end{tabular}


Table 3 Incidence of vomiting; values are number of children

\begin{tabular}{llll}
\hline & Ketamine & Midazolam & p Value \\
\hline During procedure & 0 & 2 & 0.5 \\
During recovery & 7 & 0 & 0.012 \\
At home & 4 & 2 & 0.678 \\
All individuals & 9 & 4 & 0.234 \\
\hline
\end{tabular}

${ }^{\star}$ Fisher's exact test.

questionnaire. If they were not on the phone then they were given a stamped addressed envelope with the questionnaire to complete.

- Has your child had any vomiting? If so how often and how soon after leaving hospital? When did it stop?

- Did your child experience any nightmares? If so when?

- How long did you supervise your child for after returning home?

- Was his/her walking unsteady? If so for how long?

- Was your experience in the $A \& E$ department worse, same, or better than expected?

- Does your child remember the suturing when asked? (if child 5 years or above)

The project was passed by the Lancaster, Kendal, and South Lakes research ethics committee.

Information gathered was entered onto Alpha5 a Windows '95 relational database by Dr McGlone.

\section{Results}

During the period of the study the department sutured 216 (including those in the study), stapled 94, used Steristrips on 231, and used histoacryl glue on 103 children 7 years or less. Therefore in a significant number a combination of distraction techniques and speed was utilised, sedation not being considered necessary by the clinical staff.

The two patient groups ketamine and midazolam were of similar characteristics as outlined in table 1.

Difficulty in administration of the intranasal midazolam was noted in $33(66 \%)$ children and the children remembered this unpleasant aspect of their treatment. Indeed one parent commented that she would have preferred the intramuscular route.

Two children were excluded from the study. One child could not be held securely enough to instil the midazolam intranasal drops and a

Table 4 Degree of restraint needed; values are number (\%)

\begin{tabular}{llllll}
\hline \multirow{2}{*}{ Restraint } & \multicolumn{2}{l}{ During anaesthetic } & & \multicolumn{2}{l}{ During suturing } \\
\cline { 2 - 3 } \cline { 5 - 6 } \cline { 5 - 6 } & Ketamine & Midazolam & & Ketamine & Midazolam \\
\hline 0 & $35(70)$ & $1(2)$ & & $43(86)$ & $7(14)$ \\
1 & $11(22)$ & $12(24)$ & & $5(10)$ & $16(32)$ \\
2 & $1(2)$ & $18(36)$ & & 0 & $12(24)$ \\
3 & $3(6)$ & $19(38)$ & & $2(4)$ & $15(30)$ \\
\hline
\end{tabular}

Table 5 Recovery behaviour; values are number (\%)

\begin{tabular}{lcc}
\hline Recovery behaviour & Ketamine & Midazolam \\
\hline Quiet and uneventful & $34(68)$ & $31(62)$ \\
Mild agitation & $12(24)$ & $12(24)$ \\
Moderate agitation & $3(6)$ & $4(8)$ \\
Pronounced agitation & $1(2)$ & $3(6)$ \\
\hline
\end{tabular}

child in the ketamine group had the wound sutured by a junior doctor (supervised) and this adversely effected the parental reaction and satisfaction with the procedure. One child in the ketamine group was given a further dose of $1 \mathrm{mg} / \mathrm{kg}$.

Despite apparent differences in behaviour before sedation this was not significantly different ( $p$ value 0.08 ; Mann-Whitney test). However the difference during local anaesthetic and suturing was significant, $p<0.01$ (table 2).

We strenuously followed up parents in view of ketamine's known side effect of vomiting to determine if there was a reduction (table 3 ), and all the ketamine group and all but two of the midazolam group had their questionnaires completed. In the ketamine group one child returned to the department because of repeated vomiting and two vomited during recovery and at home.

Table 4 summarises the restraint required during the local anaesthetic and suturing. Restraint was measured 0 to 3 indicating the number of body parts restrained. A MannWhitney test was used to test the null hypothesis of no difference between the two patient groups. The $p$ values of $<0.01$ in each case indicates strong evidence of a difference.

The recording of oxygen saturation did pose problems in that children who were inadequately sedated would make repeated attempts to remove the oximetry probe. The lowest oxygen saturation for the midazolam group was $86 \%$ (during a crying bout) and in the ketamine group $92 \%$. Median value $97 \%$ in both groups.

Lacrimation was noted in six of the ketamine group and 13 of the midazolam group, the latter presumably due to distress. Increased salivation was noted by the nurse in 15 ketamine and six midazolam cases, although in only one ketamine child was suctioning used. None of the doctors could recollect a case when increased secretions had posed a problem and we suspected an over-reporting of this side effect (it had been stressed in teaching to nursing staff). A transient rash occurred in five $(10 \%)$ of the ketamine group and in one of the midazolam group.

In view of the known problem of agitation and dysphoria with ketamine during recovery we were anxious to study this aspect. Table 5 summarises this behaviour. The MannWhitney test shows insufficient evidence to reject the null hypothesis that there was no difference between the groups $\mathrm{p}$ value 0.44 .

Three children in each group had a disturbed night/nightmares. However four mothers from the midazolam group stated that their children had shown aggressive behaviour at home. Indeed one mother stated that she would have preferred no sedation such was the fury of her toddler.

The time at which the child was ready for discharge (see fig 1) showed a range of times similar for both groups. However the median time was lower in the midazolam group (75 $v$ 82 minutes). 


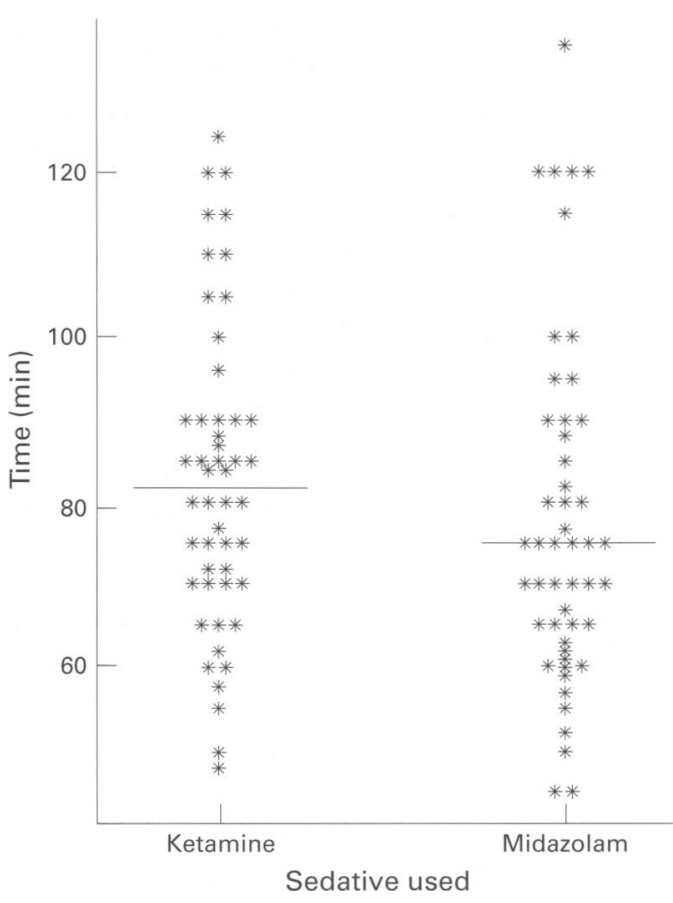

Figure 1 Time of discharge from start of procedure.

An unsteady gait after the procedure was expected, however this was sometimes difficult to quantify if the child was discharged late at night and due to go to bed. However an unsteady gait was noted in the ketamine and midazolam groups $(73 \%$ and $71 \%$ respectively) and showed no significant difference in the incidence ( $p$ value $0.77 ; \chi^{2}$ test, $1 \mathrm{df}$ ); see fig 2.

Parents showed a definite preference for sedation with ketamine (table 6 ) with a $p$ value 0.018 (Mann-Whitney). The overall satisfaction with the attendance may have been positively effected by the presence of a senior doctor. The only mother to have a negative reaction in the ketamine group had had her

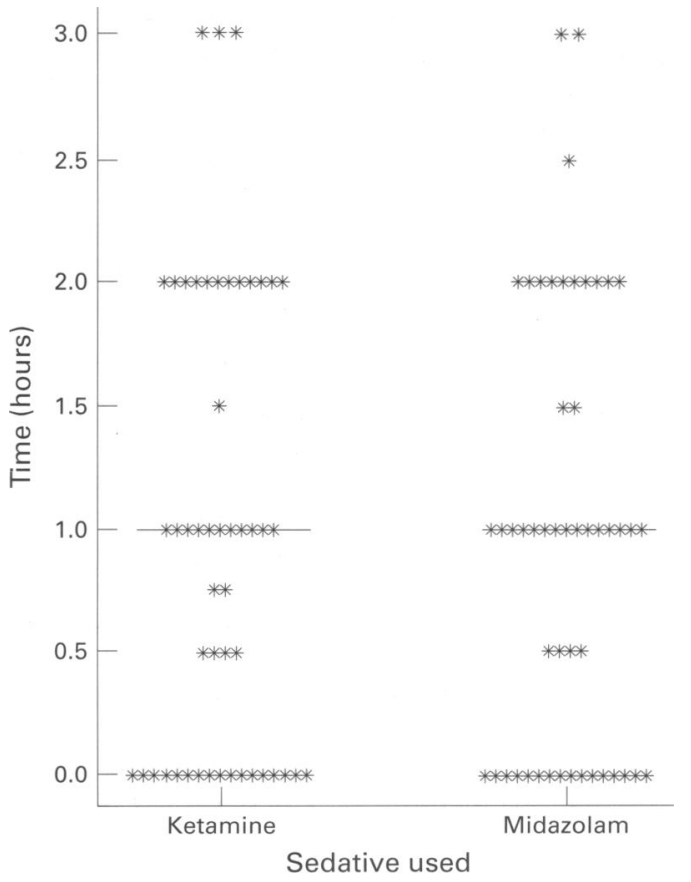

Figure 2 Period of unsteady gait after return home.
Table 6 Degree of parent satisfaction with $A \mathcal{E} E$ attendance; values are number (\%)

\begin{tabular}{lcc}
\hline Parental satisfaction & Ketamine $(n=50)$ & Midazolam $(n=48)$ \\
\hline Worse than expected & $1(2)$ & $3(6)$ \\
Same as expected & $4(8)$ & $11(23)$ \\
Better than expected & $45(90)$ & $34(71)$
\end{tabular}

child's knee wound treated with Steristrips with restraint the previous night by a senior house officer unwilling to disturb the consultant at home. During the procedure seven parents felt faint in the midazolam group and three in the ketamine group. No child of any age in either group remembered the suturing ( 30 aged 5 or above). The question regarding how long the child was supervised for was included to assess parental anxiety. The average time for the ketamine and midazolam group was 6.1 and 5.9 hours respectively. If the child was discharged late at night then the parent would often sleep with him or her.

\section{Discussion}

The search for the ideal paediatric sedative continues. Such a drug, ${ }^{6}$ would have the following properties: (1) easy and painless to administer; (2) a rapid and reliable onset of clinical action; (3) no serious side effects should be associated with its use; (4) sedation would be long enough for simple procedures, but not prolonged; and (5) cost would be reasonable in terms of the drug and manpower needed.

Midazolam has been used extensively intravenously in the $U K$ in adult $A \& E$ practice, to facilitate the reduction of fractures and dislocations.

The plasma clearance of midazolam in children is higher than in adults by a factor of two to three and this more rapid metabolic turnover of the drug should be remembered when considering the paediatric dosage. ${ }^{12}$

In UK paediatric practice the oral form of midazolam has been used. ${ }^{8}$ Flavouring and colouring is added to the intravenous preparation by the hospital pharmacy reducing the shelf life to only two weeks. Oral midazolam can usually provide some sedation within 40 minutes, ${ }^{13}$ but the increasing doses being used in studies from 0.2 to $0.5 \mathrm{mg} / \mathrm{kg},{ }^{8}{ }^{14}$ suggests it is not ideal. Oral midazolam is subject to incomplete absorption, there is a significant "first pass" metabolism with a bioavailability of $15 \%,{ }^{15}$ at higher doses and reduced gastric emptying in an anxious child may delay its effect. All of the above causes the dosing to be higher than with other routes. In view of these disadvantages its use has been abandoned in Lancaster. The bioavailabilty via the rectal route is as low as $18 \%,{ }^{15}$ and has an obvious drawback in administration to an uncooperative child. The sublingual route has been described with predictable problems of compliance in preschool children. ${ }^{16}$ The intramuscular route has been described in children ${ }^{17}$ as a premedication at a dose of $0.2 \mathrm{mg} / \mathrm{kg}$ and the bioavailability is $87 \% .{ }^{15}$ Jet injection of midazolam has been described, but in the dosage 
required for suturing $(0.2$ to $0.3 \mathrm{mg} / \mathrm{kg}$ ) would cause $67 \%$ of children to cry. ${ }^{18}$

The intranasal route has been extensively described and has been found to be safe up to a dose of $0.5 \mathrm{mg} / \mathrm{kg} .{ }^{6}{ }^{19}{ }^{20}$ In any case this would appear to be near the maximum dose in view of the volume $(5 \mathrm{mg} / \mathrm{ml})$ needed to be instilled intranasally. The bioavailability of the intranasal route has been quoted as $55 \%$ and $57 \%,{ }^{12}$ and the half life is 2.2 hours; this is similar to the intravenous half life of 2.4 hours. ${ }^{12}{ }^{21}$ The rapid onset of action (10 minutes), ${ }^{421}$ was confirmed in our study. If the doctor is to avoid the needle then the intranasal route would appear to be the obvious choice. ${ }^{22}$ However proper positioning of the child is vital in order to ensure that the full dose is given.

The major drawback to this route is the nasal irritation and discomfort caused by midazolam. ${ }^{23}$ There is of course the operational problem with this route in that some children may refuse it and possibly retch and spit it out. Acceptability can be improved by various means including the use of toys as receptacles. ${ }^{24}$ Intranasal $4 \%$ topical lignocaine spray has been used before the intranasal midazolam, but has not been subjected to any study. The irritation may be due to the preservative ( $1 \%$ benzyl alcohol), the $\mathrm{pH}$ of 3.3 , or the drug itself. Our biochemistry department attempted to buffer the drug with $0.14 \%$ sodium bicarbonate, but this resulted in precipitation at $\mathrm{pH} 4.8$. In any case the nasal absorption of a drug is related to the unionised fraction, ${ }^{25}$ and therefore if the $\mathrm{pH}$ was raised this might in itself effect the absorption.

Midazolam has a potent amnesic effect, but has no effect on memory before the drug being given. ${ }^{26}$ At a dose of $0.2 \mathrm{mg} / \mathrm{kg}$ intranasal midazolam only $29 \%$ of children undergoing dental treatment had recall. ${ }^{27}$

Intranasal midazolam has been suggested as an alternative route for the treatment of acute childhood seizures. ${ }^{28}$

Intranasal midazolam is the wrong formulation of drug via the correct route. It causes significant distress to children and until Roche provide the base drug for further research then we would not recommend this route. We are currently studying the use of intramuscular midazolam in selected children.

$\mathrm{Oral}^{7}$ and intranasal ${ }^{91029}$ ketamine has been used in children, but it has not become popular probably because of its unpleasant taste. Intravenous ketamine has been used in children in the A\&E department, ${ }^{30}{ }^{31}$ but these papers left unanswered the problem of cannulating young children. Low dose intramuscular ketamine would appear to be the ideal agent for short suturing procedures in children. The dose quoted by Green et al is $4 \mathrm{mg} / \mathrm{kg}^{1}$; at this dose supplemental local anaesthetic was used in $7.4 \%$ and further doses of ketamine in $2.8 \%$. A dose of $3 \mathrm{mg} / \mathrm{kg}$ with local anaesthetic has been shown to be effective. ${ }^{35}$ Therefore we were attempting to find out whether a lower dose could be used with perhaps a lower incidence of side effects. ${ }^{11}$

These side effects include excessive oral and tracheobronchial secretions, muscular hyper- tonicity, transient clonus, transient stridor or laryngospasm, vomiting, transient rash, unpleasant agitation, nightmares, mild respiratory depression, and apnoea. One of the most disturbing, although rare, side effects is laryngospasm. Although Green et al reported one case in a series of 108 patients $(4 \mathrm{mg} / \mathrm{kg}),{ }^{1}$ they have had three further episodes after nine years of experience of the drug (1022 cases; personal communication). The cases of laryngeal spasm needing intubation described in the world literature are rare and occur at the higher dose of $10 \mathrm{mg} / \mathrm{kg}$. ${ }^{2}$ Transient respiratory arrest has been described with rapid intravenous bolus and once via the intramuscular route, ${ }^{32} 4$ $\mathrm{mg} / \mathrm{kg}$, although it is quite possible that the latter was an inadvertent intravenous injection.

Several papers have been written suggesting guidelines for the use of low dose ketamine in the A\&E department. ${ }^{33} 34$

The incidence of vomiting varies in papers as do the dosage used and variety of procedures performed. The percentage follow up of patients at home is also variable $\left(35-71 \%^{1}\right)$ and often not quoted in other papers, so this could account for some under-reporting of this side effect. Our study did confirm that when vomiting occurred it was not during the suturing but during the recovery period. The department continued to collect data on ketamine procedures and in the next 42 cases the incidence of vomiting was one during recovery and three at home (four individuals). The median time of discharge was 100 minutes. We believe that after the results of the initial study were known nursing staff were not attempting to mobilise these children as early and this reduced the incidence of vomiting.

The average discharge time in our study of about 80 minutes is the same as that quoted by Green et al, who used a dose of $4 \mathrm{mg} / \mathrm{kg}$. ${ }^{1}$ On further inspection six of his 92 patients were discharged at 30 minutes whereas none of our patients were ready at this time. Pruitt et al using $3 \mathrm{mg} / \mathrm{kg}$ with $0.05 \mathrm{mg} / \mathrm{kg}$ midazolam had an average discharge time of 76 minutes (range 50 to 120$).^{5}$ This might demonstrate a different interpretation of the discharge criteria by nursing staff.

Muscular hypertonicity occurred in $48 \%$ in one study, ${ }^{1}$ using a dose of $4 \mathrm{mg} / \mathrm{kg}$, yet only in $2.7 \%$ in another, ${ }^{5}$ at a dose of $3 \mathrm{mg} / \mathrm{kg}$ with midazolam $(0.05 \mathrm{mg} / \mathrm{kg})$. The latter reduction was probably due to the combination of a lowered ketamine dose and the addition of midazolam. Muscular hypertonicity is dose related and has caused opisthotonus in two case reports at the high doses of 14 and 19 $\mathrm{mg} / \mathrm{kg}$ intravenous. ${ }^{2}$ Brief twitching can occur which could be confused with seizure activity, however these spasms are benign and are not associated with electroencephalographic changes. ${ }^{2}$ In our study we noted hypertonicity only on three $(6 \%)$ occasions and brief twitching occurred only on one occasion. The transient rash well recognised with ketamine occurred in five $(10 \%)$ of our children whereas at a dose of $4 \mathrm{mg} / \mathrm{kg}$ Green et al had an incidence of 19 (17.6\%). 
Finally by reading the following comments of parents from the study the need for an improved management for these children may be appreciated. "It was much better than last time (two years ago) when it was barbaric". "His brother when 4 years old was held down and was afraid of hospitals for years afterwards". "Last time they couldn't hold him still and look at the scar on his face!" To ensure against any complacency please note that these comments relate to other hospitals, although we had similar statements about our own department.

\section{Conclusion}

Low dose intramuscular ketamine would appear to be the drug of choice for suturing of young children in the A\&E department. However in view of the potential problems the drug must be restricted to use by doctors trained in emergency paediatric airway management and resuscitation either from an $A \& E$ or anaesthetics background. More research needs to be done with even lower dose regimens which might reduce the incidence of side effects and shorten the recovery time further. The greatest risk of this drug is complacency and the potential for indiscriminate use, which could develop once more doctors become familiar with its obvious benefits.

We would like to thank Mr M Brogan, Head Biomedical Scientist, Biochemistry, Royal Lancaster Infirmary; Ms S Hollis, Statistics Department, Lancaster University; and Mr M Flowers Retired A\&E Consultant at Leeds General Infirmary who originally suggested an investigation of ketamine in children.

1 Green G, Nakamura R, Johnson E. Ketamine sedation for paediatric procedures: part 1, a prospective series. An Emerg Med 1990;19:1024-32.

2 Green G, Johnson E. Ketamine sedation for paediatric procedures: part 2, review and implications. Ann Emer Med 1990;19:1033-46.

3 Epstein F. Ketamine dissociative sedation in paediatric emergency medical practice. Am J Emerg Med 1993;11 $180-2$.

4 Theroux M, West D. Efficacy of intranasal midazolam in facilitating suturing of lacerations in preschool children in the emergency department. Pediatrics 1993;91:624-7.

5 Pruitt J, Goldwasser M. Intramuscular ketamine, midazolam, and glycopyrrolate for paediatric sedation in the emergency department. J Oral Maxillofac Surg 1995;53: 13-17.

6 Yealy D, Ellis J. Intranasal midazolam as a sedative for children during laceration repair. Am J Emerg Med 1992;10: 584-7.

7 Qureshi F, Mellis P. Efficacy of oral ketamine for providing sedation and to children requiring laceration repair. Pediatr

8 Connors K, Terndrup T. Nasal versus oral midazolam for sedation of anxious children undergoing laceration repair. Ann Emerg Med 1994;24:1074-9.
9 Louon A, Reddy V. Nasal midazolam and ketamine for paediatric sedation during computerised tomography. Acta Anaesthesiol Scand 1994;38:259-61.

10 Abrams R, Morrison J. Safety and effectiveness of intranasal administration of sedative medications (ketamine, midaadministration of sedative medications (ketamine, midacedures. Anaesthesia Progress 1993;40:63-6.

11 Huwitz G, DeVore D. Decreased doses of ketamine hydrochloride in children: a triple blind study. Journal of the Baltimore College of Dental Surgery 1975;30:28-34.

12 Rey E, Delaunay L. Pharmacokinetics of midazolam in children: comparative study of intranasal and intravenou administration. Eur J Clin Pharmacol 1991;41:355-7.

13 Walker J. The use of oral midazolam in A\&E to reduce anxiety in children. Accid Emerg Nursing 1996;4:110-13.

14 Taiwo B, Flowers M, Zoltie N. Reducing children's fear when undergoing painful procedures. Arch Emerg Med 1992;9:306-9.

15 Payne JG, Mattheyse FJ. The pharmacokinetics of midazolam in paediatric patients. Eur J Clin Pharmaco 1989;37:267-72.

$16 \mathrm{Karl} \mathrm{H}$, Rosenberger J. Transmucosal administration of midazolam for premedication of pediatric patients. Anesthesiology 1993;78:885-91.

17 Taylor MB, Vine PR. Intramuscular midazolam premedication in young children. Anaesthesia 1986;41:21-6.

18 Greenberg RS, Maxwell LG. Preanaesthetic medication of children with midazolam using the Biojector jet injector. Anesthesiology 1995;83:264-9.

19 Bozkurt P, Kilic N. The effects of intranasal midazolam on urodynamic studies in children. Br J Urol 1996;78:282-6.

20 Fosel T. Hack C, Knoll R. Nasal midazolam in children, plasma concentrations and the effect on respiration. Paediatr Anaesth 1995;5:347-53.

21 Malinovsky J, Lejus C, Servin F. Plasma concentrations of midazolam after IV, nasal or rectal administration in children. Br J Anaesth 1993;70:617-20.

22 Fraser G. Intranasal midazolam. Hospital Pharmacy 1992; 27:73-4.

23 Lugo R, Fishbein M. Complication of intranasal midazolam [letter]. Pediatrics 1993;92:638.

24 Cohen $M$, Gur E. Intranasal administration of midazolam with a dinosaur toy [letter]. Plast Reconstr Surg 1995;95 421-2.

25 Sakane T. Direct drug transport from the rat nasal cavity to the cerebrospinal fluid: the relation to the dissociation of the drug. I Pharm Pharmacol 1994;46:378-9.

26 Twersky R, Hartung J. Midazolam enhances anterograde but not retrograde amnesia in paediatric patients. Anaesthesiology 1993;78:51-5.

27 Kupietzky A, Holan G. Intranasal midazolam better at effecting amnesia after sedation than oral hydroxyzine: a pilot study. Pediatr Dent 1996;18:32-4.

28 Wallace S. Nasal benzodiazepines for management of acute childhood seizures? Lancet 1997;349:222.

29 Aldrete J, Russell L, Davis F. Intranasal administration of ketamine: possible applications. Acta Anaesthiol Belg (Suppl) 1988;39:95-6.

30 Dachs RJ, Innes GM. Intravenous ketamine sedation of paediatric patients in the emergency department. Ann paediatric patients in the eme

31 Parker RI, Mahan RA. Efficacy and safety of intravenous midazolam and ketamine as sedation for therapeutic and diagnostic procedures in children. Pediatrics 1997;99:427 31.

32 Smith J, Santer L. Respiratory arrest following intramuscular ketamine injection in a 4 year old child. Ann Emer Med 1993;22:613-15. [Letters 1993;23:139-40.]

33 Duda JThe good, bad, and ugly: using ketamine for ED pediatric patients. J Emerg Nurs 1996;22:49-51.

34 Glickman A. Ketamine: the dissociative anesthetic and the development of a policy for its safe administration in the pediatric emergency department. J Emerg Nurs 1995;21: 116-24. 